\title{
Experimental observation for dynamic characteristics of "Shimajiri Mahji" and its evaluation using computational simulation
}

\author{
Y Higa'*, H lyama², K Shimojima', O Higa ${ }^{3}, \mathbf{S}$ Itoh $^{4}$ \\ 1. Department of Mechanical Systems Engineering, National \\ Institute of Technology (KOSEN), Okinawa College, Japan \\ 2. Department of Mechanical \& Intelligent Systems \\ Engineering, National Institute of Technology (KOSEN), \\ Kumamoto College, Kumamoto, Japan \\ 3. Science \& Technology Division, National Institute of \\ Technology (KOSEN), Okinawa College, Japan \\ 4. Institute of Shockwave Advanced Technology (ISAT), \\ Kumamoto, Japan
}

\begin{abstract}
We perform herein a direct observation of shockwave propagation using a high-speed camera and estimate properties, such as acoustic velocity and Hugoniot parameter, using the impedance matching scheme to reveal the dynamic characteristics of Okinawa's unique soil "Shimajiri Mahji." A computational simulation corresponding to the experimental setup is then conducted. A comparison of the numerical and experimental results demonstrates their fairly good agreement, which suggests that the experimentally estimated dynamic characteristics of "Shimajiri Mahji" are valid. In addition, a computational simulation based on the smoothed particle hydrodynamics is also modeled and performed to elucidate the fragment behavior when the unexploded bomb explosive is at the soil surface. From a series of computational results, we have confirmed and clarified that the fragment behavior is significantly dependent on the amount of explosive charge and soil characteristics.
\end{abstract}

\section{INTRODUCTION}

To contribute to the development of the safety improvement processing technology used for unexploded bomb disposal from the viewpoint of computational mechanics, this study aims to (1) set a theoretical evacuation area and (2) aggressively reduce the evacuation area. Accordingly, a simple simulator associated with the underground and ground surface explosion problem is constructed for the former. The shockwave propagation behavior and the crater formation related to the explosive amount and buried depth are also reported [1]. The computational modeling based on the smoothed particle hydrodynamics scheme is constructed [2,3], and the fragment behaviors are observed to visualize the fragment behaviors generated during the unexploded bomb blasting with a particle image. For the latter, the evacuation area could be positively reduced by examining the control effect of the fragment

*Corresponding Author: y.higa@okinawa-ct.ac.jp 
behaviors depending on the design of the treatment trench by linear plate construction and its exit shape [4,5]. Meanwhile, the dynamic characteristics of Okinawa's unique soils, "Jahgaru," are revealed by calculating the material parameters through an examination of the direct observation using a high-speed camera of how the shockwaves induced by detonation are incident, transmitted, and reflected in the soil. The soil dynamic characteristics were identified by comparing and verifying these experimental systems with a similar type of numerical simulation [6]. "Shimajiri Mahji," which is the target of this report, is a reddishbrown soil with Ryukyu limestone as the formation phase and widely distributed in the southern area of the main island of Okinawa Prefecture. Its generation and soil taxonomical considerations have been reported in the past $[7,8]$. However, no one has reported on the dynamic characteristics describing the high-speed deformation associated with the unexploded explosive explosion phenomenon in our research reviews. Therefore, the dynamic characteristics of "Shimajiri Mahji" essential in contributing to the safety processing technology for unexploded bomb disposal are clarified herein. Moreover, the visualization of the experimental systems and the verification of the validity of the dynamic characteristics are performed by creating a numerical simulation model that reproduces the system of visualization experiments [9-13] and by performing numerical simulations introducing the dynamic characteristics of soil calculated from the experimental results.

\section{EXPERIMENTAL PROCEDURE AND ITS RESULT}

We targeted Okinawa's unique soil "Shimajiri Mahji" and obtained undisturbed samples from a point that was $0.50 \mathrm{~m}$ deep from the ground surface using the thin wall tube sampling method. A $5 \mathrm{~mm}$-thick $50 \mathrm{~mm}^{2}$ soil sample was cut and used as the sample for the dynamic characteristic evaluation. Figure 1 shows that blasting with a detonation fuse was performed after preparing the sample between acrylic resin blocks (PMMA: polymethyl methacrylate) and after installing the explosive lens consisting of SEP and HABW fixed on the upper part by the PV tube. Consequently, a planar shock wave induced by the detonation was incident on the soil. During the series of processes, incident/reflected and reflected/transmitted waves were generated at the interfaces between PMMA and the object and between the object and PMMA from the top of the drawing, respectively. The shock waves passing through PMMA were imaged using Schlieren's optical observation method. Figure 2 presents the experimental device for the optical observation. Tables 1 and 2 show the high-speed camera and pulse laser specifications, respectively. The experimental movie simultaneously started shooting as soon as the electricity flowed in the electric detonator. The shock wave velocity entering the soil was controlled by parametrically changing the thickness of the upper PMMA to $t=20,30$, and $40 \mathrm{~mm}$. As an example of the experimental results, Fig. 3 shows a snapshot of the optical observation video associated with $t=40 \mathrm{~mm}$. The plane shock wave generated by the explosive lens entered PMMA from the top, and the wave front edge was transmitted in PMMA. Subsequently, the shock wave propagation and the front are observed as follows: (1) $\tau=17.8 \mu \mathrm{s}$, just before entering the PMMA-Shimajiri Mahji interface, (2) $20.0<\tau<22.0 \mu \mathrm{s}$, which propagated through the soil, and (3) $\tau=23.2 \mu \mathrm{s}$, which was incident on the PMMA side from the Shimajiri Mahji-PMMA interface. We can calculate the transmitted shock wave velocity depending on the difference in the incident shock wave velocity by analyzing the relationship between the time of the shock wave leading edge and the movement distance from these observation videos. The unknown shock wave propagation velocity immediately 


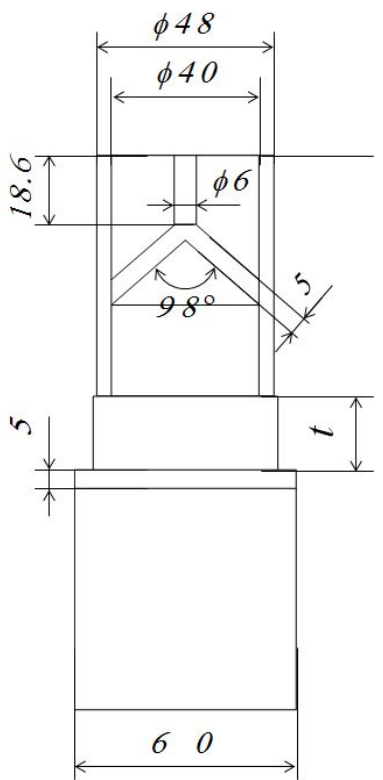

Dimension of the experimental device

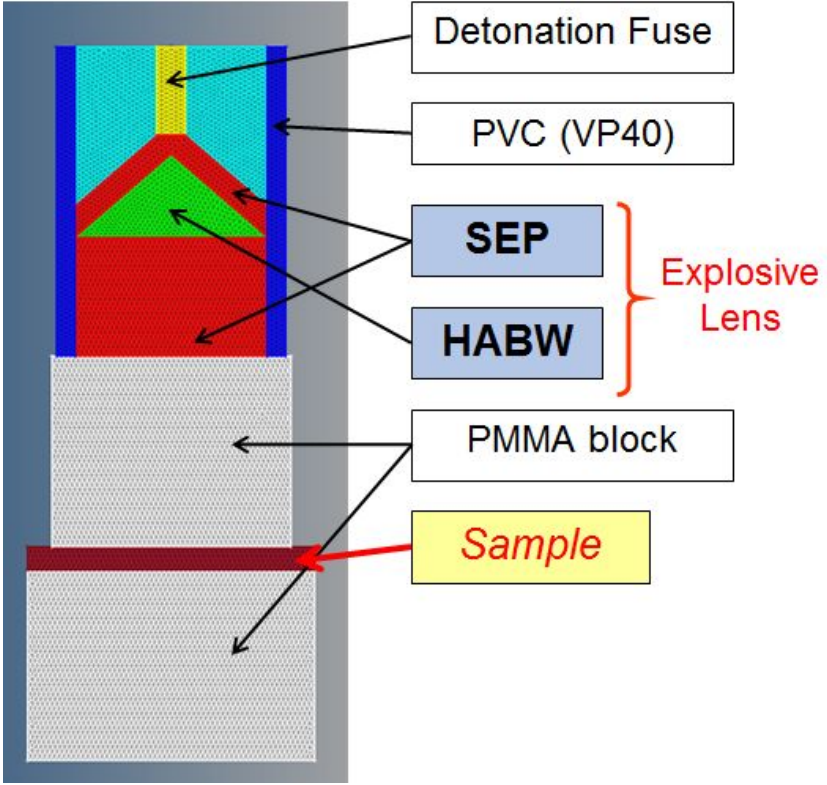

Schematic diagram of the device

Figure 1 Dimensions and schematic diagram of the experimental device

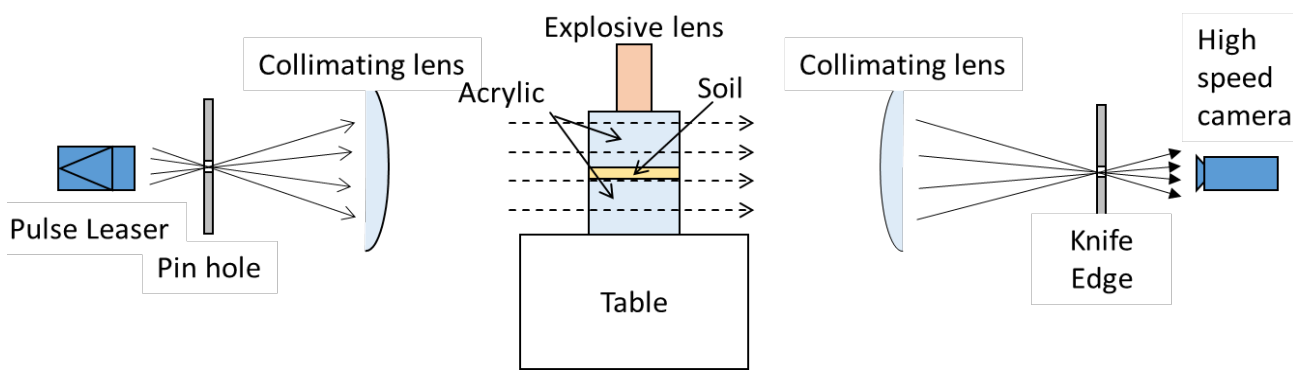

Figure 2 Optical layout of the Schlieren system

before the incidence and immediately after the permeation was determined from the shock wave propagation velocity of the known-quantity PMMA using the nonlinear curve fitting method [14]. From the experimental data, we calculated the shock wave velocity and the particle velocity of Shimajiri Mahji using the impedance matching method [15]. Furthermore, we obtained the shock wave propagation velocity $C_{0}$ and Hugoniot coefficient $s$, which are the dynamic characteristics unique to the soil, by plotting the relationship between $U_{s}$ and $U_{p}$. The experimental data showed that by increasing the PMMA thickness, the particle velocity can be reduced by damping the generated shock wave. The shock wave velocity was also calculated for all the experimental movie data, with the results summarized in Table 3. Figure 4 depicts the data of 11 points in Table 3 related to the shock wave velocity, $U_{s}^{\prime}$ and particle velocity, $u_{p}$ (diagram). As in the previous report [6], the experimental points can be 
approximated by a linear line. From the abovementioned findings, the shock wave propagation velocity $C_{0}$ and the Hugoniot curve coefficient $s$, which are the dynamic characteristics specific to Shimajiri Mahji, were obtained as $C_{0}=2.57 \mathrm{~km} / \mathrm{s}$ and $s=1.73$, respectively.

Table 1 High-speed video camera specification.

\begin{tabular}{ll}
\hline Product name & Kirana \\
Resolution & $924 \times 768 \mathrm{px}$ \\
Continuous recording capacity & 180 frames max. \\
Frame rate & $5 \mathrm{Mfps}$ \\
\hline
\end{tabular}

Table 2 Pulse laser specification.

\begin{tabular}{ll}
\hline Model number & SI-LUX640 \\
Output & $200 \mathrm{~W}$ \\
Wavelength & $640 \mathrm{~nm}$ \\
\hline
\end{tabular}

Table 3 Experimental results for Shimajiri Mahji.

\begin{tabular}{|c|c|c|c|}
\hline $\begin{array}{l}\text { PMMA } \\
\text { gap } t, \mathrm{~mm}\end{array}$ & $\begin{array}{l}\text { PMMA -> Shimajiri Mahj } \\
\text { Incident velocity } U_{s}, \mathrm{~km} / \mathrm{s}\end{array}$ & $\begin{array}{l}\text { Shimajiri Mahji -> PMMA } \\
\text { Transmitted velocity } U_{s}^{\prime}, \mathrm{km} / \mathrm{s}\end{array}$ & $\begin{array}{l}\text { Particle velocity } \\
U_{p}, \mathrm{~km} / \mathrm{s}\end{array}$ \\
\hline \multirow{3}{*}{40} & 3.53 & 3.47 & 0.47 \\
\hline & 3.39 & 3.34 & 0.41 \\
\hline & 3.45 & 3.38 & 0.45 \\
\hline \multirow{4}{*}{30} & 3.55 & 3.49 & 0.50 \\
\hline & 3.62 & 3.55 & 0.54 \\
\hline & 3.84 & 3.77 & 0.66 \\
\hline & 3.89 & 3.81 & 0.67 \\
\hline \multirow{4}{*}{20} & 4.01 & 3.93 & 0.75 \\
\hline & 3.70 & 3.62 & 0.57 \\
\hline & 3.83 & 3.73 & 0.65 \\
\hline & 3.53 & 3.50 & 0.49 \\
\hline
\end{tabular}

Table 4 Material constants in the constitutive equation.

\begin{tabular}{llll} 
& PVC & PMMA & Shimajiri Mahji \\
\hline Yield stress $a, \mathrm{MPa}$ & $1.0 \mathrm{e}+35$ & $1.0 \mathrm{e}+35$ & 0.120 \\
Hardening coefficient $b, \mathrm{MPa}$ & 0 & 0 & 0 \\
Strain hardening exponent $n$ & 1 & 1 & 0.01 \\
Strain rate constant $c$ & 0 & 0 & 0 \\
Young Modulus $E, \mathrm{GPa}$ & 3.3 & 2.7 & $12.3 \mathrm{e}-3$ \\
Poisson's ratio $v$ & 0.33 & 0.33 & 0.495 \\
\hline
\end{tabular}

Table 5 Material constants in the Mie-Grüneisen EOS.

\begin{tabular}{lllll}
\hline & $\boldsymbol{\rho}_{\mathbf{0}}, \mathbf{k g} / \mathbf{m}^{\mathbf{3}}$ & $\boldsymbol{C}_{\mathbf{0}}, \mathbf{m} / \mathbf{s}$ & $\boldsymbol{s}$ & $\boldsymbol{\Gamma}$ \\
\hline PVC & 1380 & 2300 & 1.47 & 0.4 \\
PMMA & 1180 & 2260 & 1.82 & 1.5 \\
Shimajiri Mahji & 1740 & 2570 & 1.73 & 075 \\
\hline
\end{tabular}




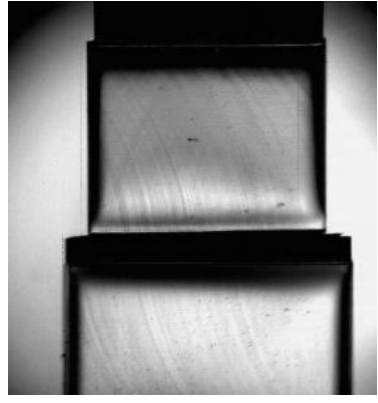

$\tau=6.2 \mu \mathrm{s}$

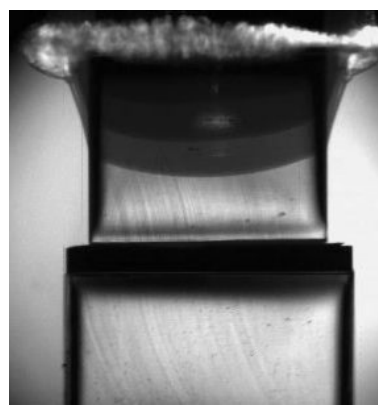

$\tau=14.0 \mu \mathrm{S}$

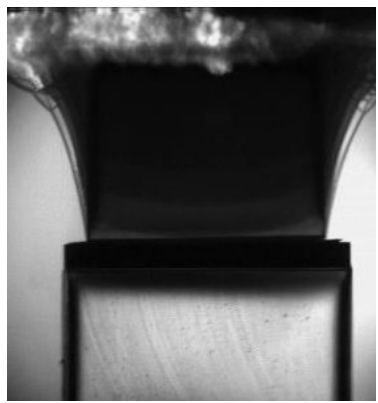

$\tau=20.0 \mu \mathrm{s}$

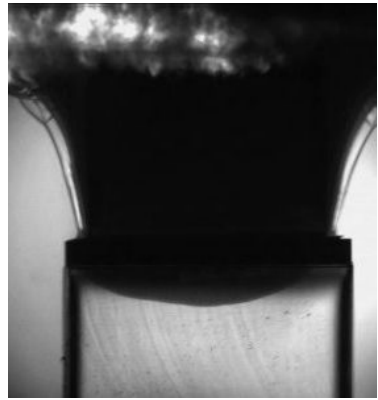

$\tau=23.4 \mu \mathrm{s}$

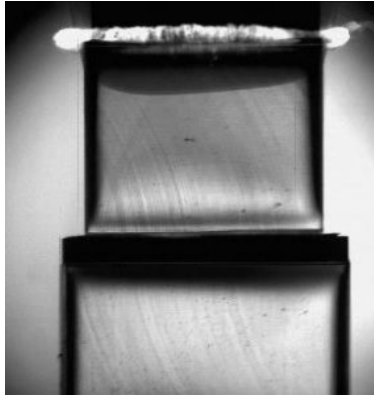

$\tau=10.0 \mu \mathrm{s}$

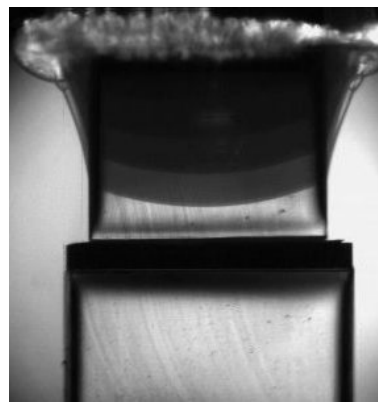

$\tau=16.0 \mu \mathrm{S}$

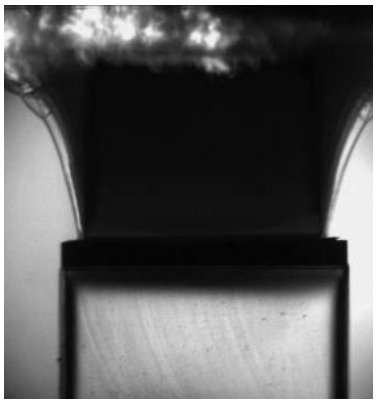

$\tau=22.0 \mu \mathrm{s}$

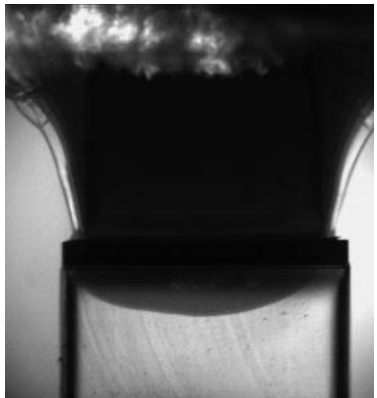

$\tau=24.0 \mu \mathrm{s}$

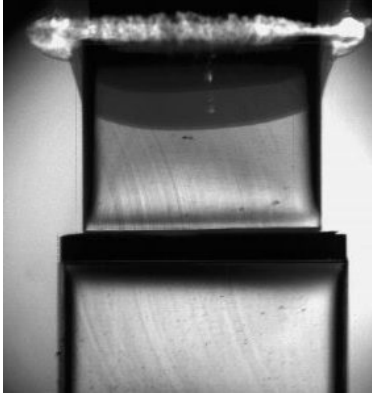

$\tau=12.0 \mu \mathrm{s}$

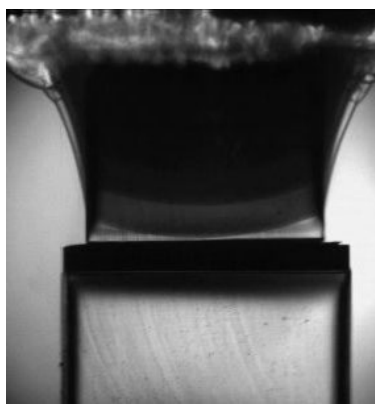

$\tau=17.8 \mu \mathrm{S}$

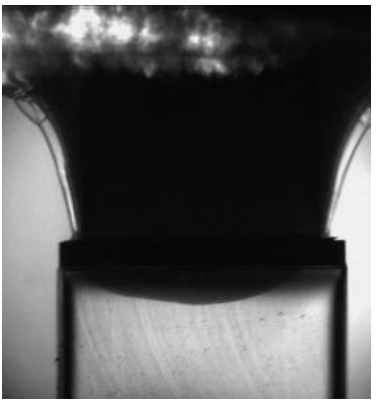

$\tau=23.2 \mu \mathrm{s}$

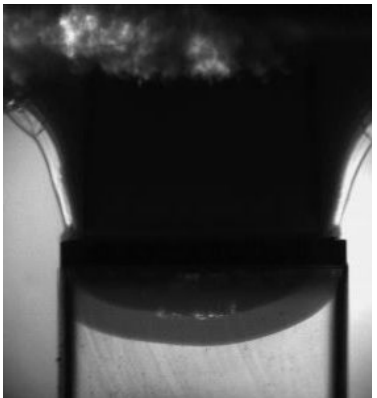

$\tau=26.0 \mu \mathrm{s}$

Figure 3 Snapshots of the optical observation result with respect to the PMMA thickness $t=40 \mathrm{~mm}$. 


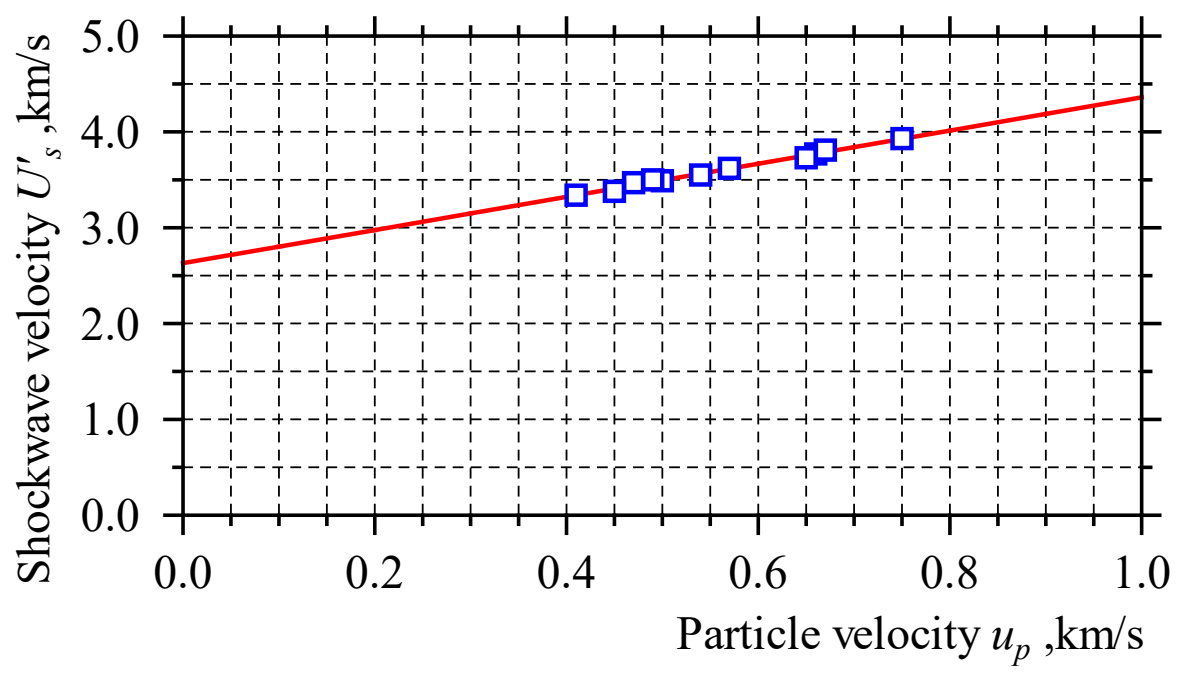

Figure 4 Hugoniot EOS of "Shimajiri Mahji"

\section{COMPUTATIONAL MODELING OF THE EXPERIMENTAL SETUP}

In the experimental method discussed in the previous section, the explosion phenomena in the explosive lens and the large deformation behavior for PMMA, PVC, and Shimajiri Mahji must be dealt with. In other words, modeling the multiphysics phenomena and its handling is indispensable. Therefore, we constructed herein a three-dimensional analysis model of the experimental system using HyperWorks-RADIOSS (® Altair), which is a commercial finite element analysis software. The quarter model (Fig. 5) was the analysis target, and the PVC, SEP, HABW, PMMA, and Shimajiri Mahji were discretized with the tetrahedral element for the Arbitrary Lagrangian Eulerian (ALE) computation because of the symmetry of the problem [16]. The total number of nodes and elements in the model shown in Fig. 5, where the PMMA thickness was $40 \mathrm{~mm}$, was 58,548 nodes and 293,666 elements, respectively.

\subsection{Equation of state and constitutive equation}

In this study of experimental observation, the explosive lens, PMMA, and soil are easily understood to immediately collapse with a fragment behavior like fluid. Therefore, the materials (Fig. 5) were assumed to be those materials obeying the Johnson-Cook's type of constitutive equation (1) and Mie-Grüneisen equation of state (2) presented as follows $[17,18]$ :

$$
\begin{gathered}
\sigma=\left(a+b \varepsilon^{n}\right)\left(1+c \cdot \log \frac{\dot{\varepsilon}}{\dot{\varepsilon_{0}}}\right) \\
P=\frac{\rho_{0} C_{0}^{2} \eta}{(1-s \eta)^{2}}\left[1-\frac{\Gamma_{0} \eta}{2}\right]+\Gamma_{0} \rho_{0} e, \quad \eta=1-\frac{\rho_{0}}{\rho}
\end{gathered}
$$

where, $\sigma, \varepsilon$, and $\dot{\varepsilon}$ are the material stress, strain, and strain rate, respectively, and $a, b, c, n$, and $\dot{\varepsilon}_{0}$ are the material constants associated with PMMA, PVC, and Shimajiri Mahji. We assumed herein that PMMA and PVC are elastic materials, and soil is an elastic-plastic associated with an independent strain rate (Table 4). In Eq. (2), $P, \rho_{0}$, and $e$ are the pressure, initial density, 
and initial energy per unit reference volume, respectively. $C_{0}, s$, and $\Gamma_{0}$ are the bulk speed of sound, linear Hugoniot slope coefficient, and Grüneisen's gamma at the reference state, respectively (Table 5).
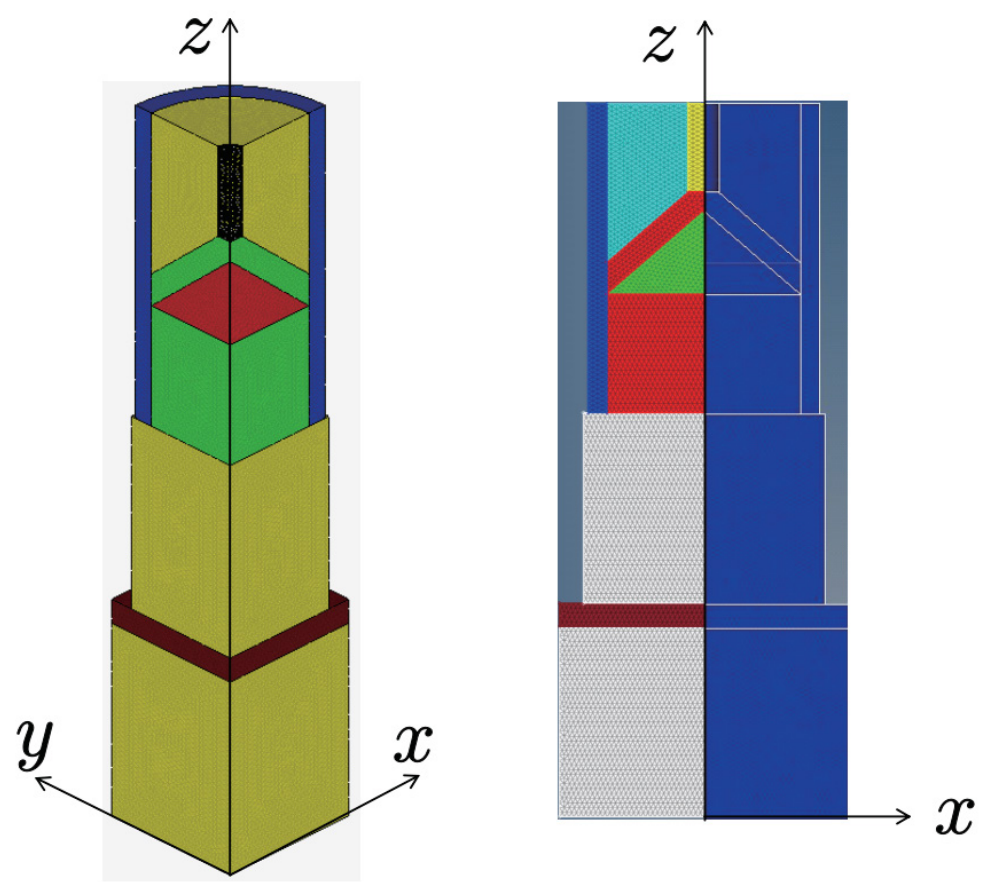

Figure 5 Birdview (left) and cross-section (right) of the computational model associated with the experimental setup.

\subsection{Explosive lens (SEP and HABW)}

The pressure generated by the expansion of the detonation products of the chemical explosive is defined by the Jones-Wilkins-Lee (JWL) equation of state [19]. The JWL equation of state defines the pressure $P_{J W L}$ as follows:

$$
\begin{gathered}
P_{J W L}=A\left[1-\frac{\omega}{R_{1} V}\right] \mathrm{e}^{-R_{1} V}+B\left[1-\frac{\omega}{R_{2} V}\right] \mathrm{e}^{-R_{2} V}+\frac{\omega E}{V} \\
P_{C J}=\frac{\rho_{0} D^{2}}{\gamma+1}, \quad \gamma=\frac{c_{p}}{c_{v}}
\end{gathered}
$$

where $A, B, R_{1}, R_{2}$, and $\omega$ are the material constants; $V$ is the relative volume of the detonation product; $E$ is the detonation energy per unit volume with an initial value of $E_{0} ; P_{C J}$ indicates the Chapman-Jouguet pressure depending on the initial density $\rho_{0}$ of the explosive; and the detonation velocity $D$ and $\gamma$ indicate the ratio of specific heat at constant volume, $c_{v}$, and at constant pressure, $c_{p}$, respectively. Table 6 provides the JWL parameters of SEP and HABW [4]. 


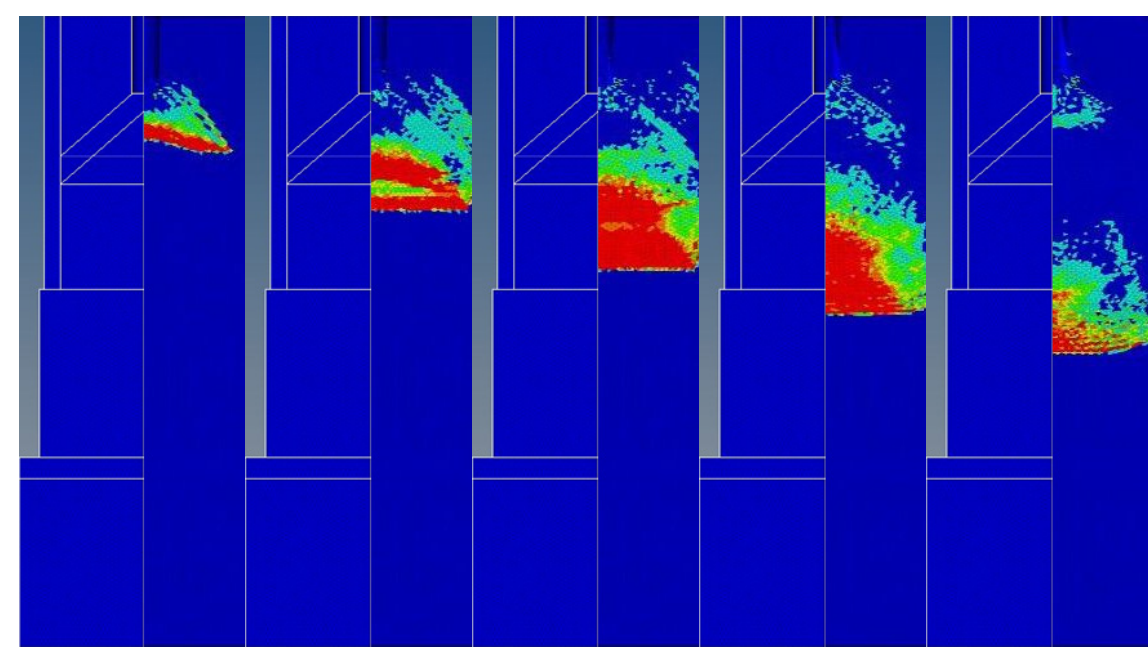

$\tau=2.0 \mu \mathrm{s}$

$\tau=4.0 \mu \mathrm{s}$

$\tau=6.0 \mu \mathrm{s}$

$\tau=8.0 \mu \mathrm{s}$

$\tau=10.0 \mu \mathrm{s}$
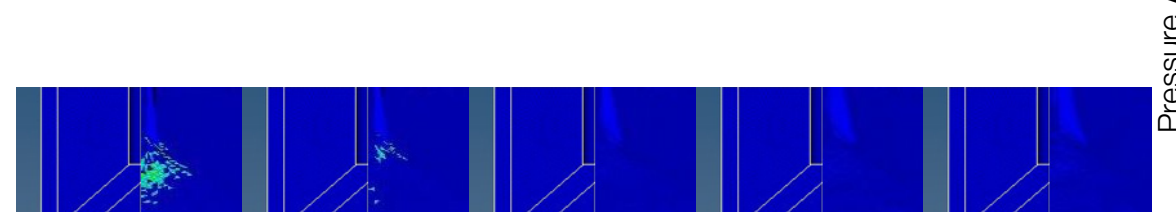

2.50
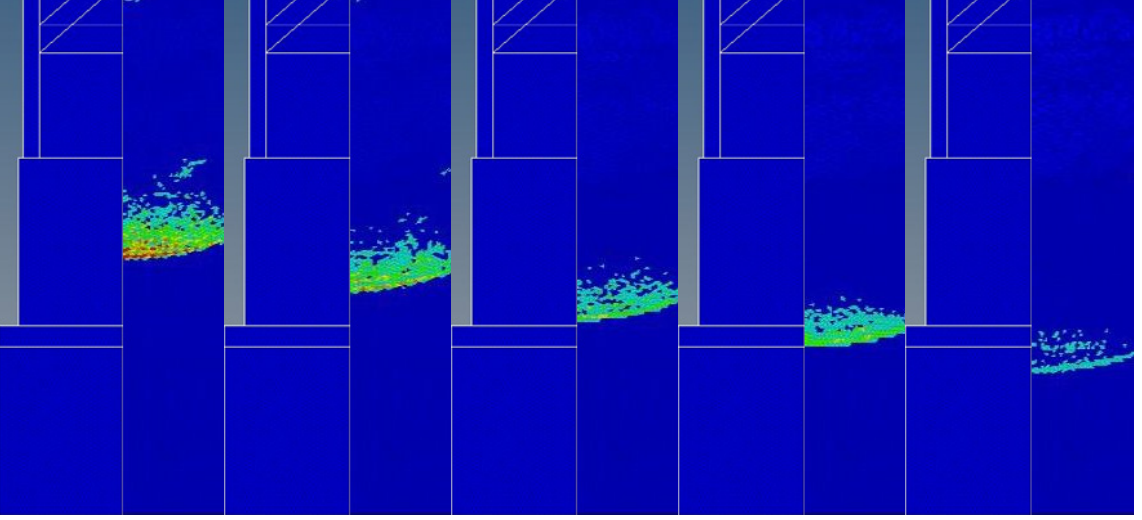

$\frac{0}{0}$

ڤ్

1.67

0.83

$\tau=12.0 \mu \mathrm{s} \quad \tau=14.0 \mu \mathrm{s} \quad \tau=16.0 \mu \mathrm{s} \quad \tau=18.0 \mu \mathrm{s} \quad \tau=20.0 \mu \mathrm{s}$

Figure 6 Snapshots of the pressure propagation behavior at each time step. This result corresponds to the PMMA gap length $t=40 \mathrm{~mm}$. 
Table $6 \mathrm{JWL}$ parameters for the explosive lens.

\begin{tabular}{lllllllll}
\hline & $\boldsymbol{P}_{\boldsymbol{C J}}, \mathbf{G P a}$ & $\boldsymbol{D}, \mathbf{m} / \mathbf{s}$ & $\boldsymbol{\rho}_{\mathbf{0}}, \mathbf{k g} / \mathbf{m} \mathbf{3}$ & $\boldsymbol{A}, \mathbf{G P a}$ & $\boldsymbol{B}, \mathbf{G P a}$ & $\boldsymbol{R}_{\mathbf{1}}$ & $\boldsymbol{R}_{\mathbf{2}}$ & $\boldsymbol{\Omega}$ \\
\hline $\mathbf{H A B W}$ & 12.4 & 4750 & 2200 & 835 & 12.67 & 5.85 & 1.53 & 0.247 \\
$\mathbf{S E P}$ & 15.9 & 6980 & 1310 & 365 & 2.31 & 4.3 & 1.0 & 0.28 \\
\hline
\end{tabular}

Table 7 Peak pressure at PMMA to Shimajiri Mahji (black $x$-mark) and Shimajiri Mahji to PMMA (red x-mark, as shown in Fig. 7).

\begin{tabular}{lll}
\hline & Comp. result, GPa & Exp. result, GPa \\
\hline PMMA -> Shimajiri Mahji (black x-mark) 2.71 & 2.60 \\
Shimajiri Mahji -> PMMA (red x-mark) & 1.84 & 2.24 \\
\hline
\end{tabular}

\section{COMPUTATIONAL RESULTS AND DISCUSSIONS}

A computational model for the experimental system was developed (Fig. 3), and a computational simulation introducing the Shimajiri Mahji's material parameters was performed to verify the validity of the dynamic characteristics shown in the previous chapter. Figure 6 shows a snapshot of the pressure propagation behavior-transmitting shockwave from PMMA to soil, incidenting to soil, and reincidenting from soil to PMMA. A planar shock wave induced by the explosive lens detonation propagating in PMMA was confirmed and reached the interface between PMMA and Shimajiri Mahji. Figure 7 indicates the pressure history immediately after the incident and transmission into Shimajiri Mahji. The black and red x-marks correspond to the point from PMMA to soil and from soil to PMMA, respectively. A primary peak pressure was generated at these points by the arrival of the plane shock wave. Subsequently, several hundred nanoseconds later, a second peak pressure depending on the difference in the shock impedance was also observed. Table 7 summarizes the calculation and experimental results at the points corresponding to two points in Fig. 7. Here the experimental result was the pressure calculated from the Hugoniot equation of state $[15,19]$. These results suggest that the dynamic characteristics of Shimajiri Mahji estimated from the experimental outcomes were fairly valid, although the peak pressure after permeation was estimated to be small.

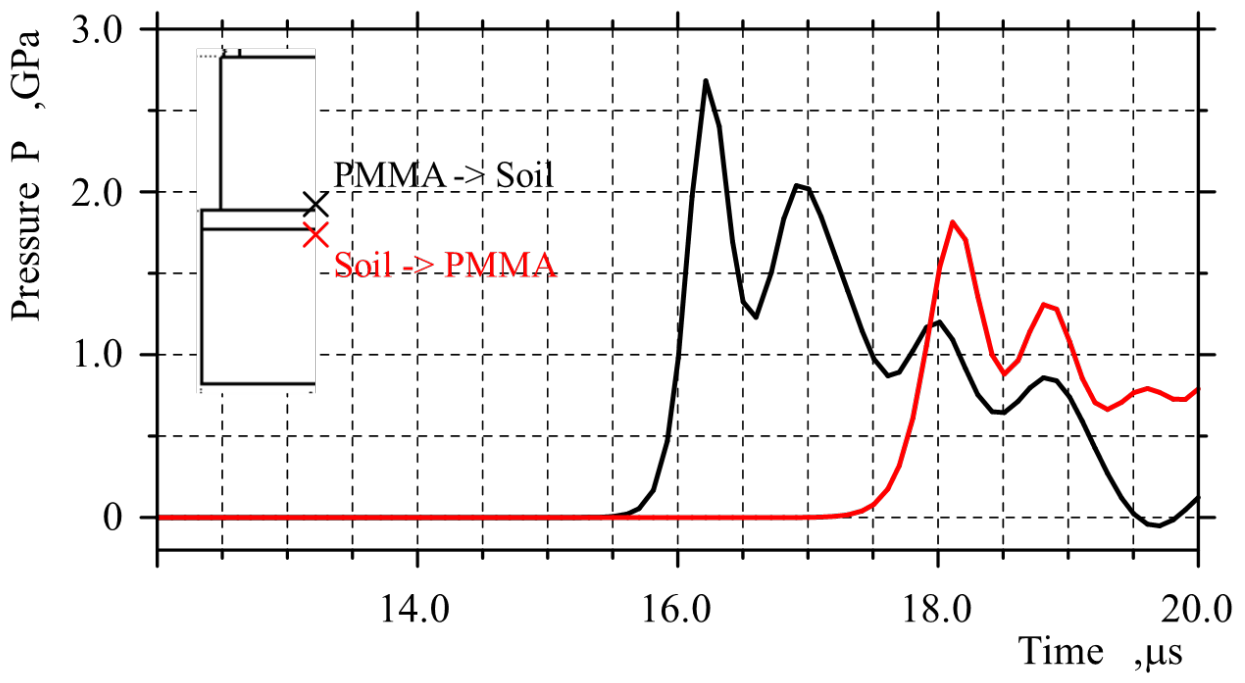

Figure 7 Pressure vs. time history at the point of PMMA to soil and soil to PMMA 


\section{CONCLUSIONS}

This study conducted an optical observation to investigate dynamic properties, such as shockwave propagation, pressure, and particle velocity, using a high-speed camera and shock characteristics using the impedance matching method to reveal the shock characteristics of Okinawa's unique soils "Shimajiri Mahji." A computational model for the experimental procedure using commercial FEM software was also developed to clarify the validity of the material characteristics. A comparison between the numerical and experimental results showed that the computational results were fairly in good agreement with the experimental results, leading to the suggestion that the dynamic characteristics of Shimajiri Mahji estimated from the experimental outcomes is quite valid.

The dynamic characteristics of the soil identified in this study are significantly important data for the safety disposal process of an unexploded bomb. The computational simulation of the fragment behavior depending on the charge of the explosive, bomb shape, and soil characteristics is now in progress.

\section{ACKNOWLEDGMENT}

The authors gratefully acknowledge financial support from JSPS KAKENHI Grant-in-Aid for Scientific Research (C), 16K01305 and 19K12393. The experiment was conducted with the help of Prof. Shigeru TANAKA and the Explosion Experiment Facilities in the Institution of Pulsed Power Science, Kumamoto University. We would also like to thank the joint research and the relationship. Moreover, the authors would like to express their sincere thanks to Mr. Asahi GENKA and Mr. Shotaro UEHARA for their tremendous effort in computational simulation.

\section{REFERENCES}

[1] Y. Higa, K. Uehara, H. Iyama, T. Tamaki and S. Itoh; A Computational Simulation for Explosive Ordnance Disposal, Materials Science Forum, Vol.767 (2014), pp.74-79.

[2] R.A. Gingold and J.J. Monaghan; Smoothed particle hydrodynamics: theory and application to non-spherical stars, Monthly Notices Royal Astronomical Society, Vol.181 (1977) pp.375-389

[3] L.B. Lucy; A numerical approach to the testing of the fission hypothesis, Astronomical Journal, Vol.82 (1977), pp.1013-1024

[4] Y. Higa, H. Iyama, K. Shimojima, O. Higa and S. Itoh; Numerical Simulation for Soil Surface Explosion Problem, - A study of fragments controlling effect using liner plate application -, The 3rd International Conference on Engineering Science and Innovative Technology (ESIT2018)

[5] Y. Higa and H. Iyama; Unexploded Bomb Safely Disposal based on the Computational Mechanics Viewpoint: Numerical Simulation for Underground/Soil Surface Explosion Problem, J. Japan Society for Design Engineering, Vol.54, No.2 (2019), pp.91-97. (in Japanese)

[6] Y. Higa, H. Iyama, K. Shimojima, M. Nishi and S. Itoh; Experimental Study and Computational Simulation for Shock Characteristics Estimation of Okinawa's Soils "Jahgaru", The International Journal of Multiphysics, Vol.11, No.3 (2017), pp.245-253.

[7] Y. Tokashiki; The characteristic properties of the Shimajiri Mahji and Jahgaru soils in Okinawa prefecture, Pedologist, Vol.37, No.2 (1993), pp.99-112. 
[8] Y. Maejima; Pedogenesis and classification of Kunigami Mahji, Shimajiri Mahji, and Jahgaru soils distributed in the subtropical islands, Japan, Pedologist, Vol.60, No.1 (2016), pp.65-70.

[9] S. Itoh, Z. Liu and Y. Nadamitsu; An investigation on the properties of underwater shock wave generated in underwater explosive of high explosive, ASME J. Pressure Vessel Technol. Vol.119 (1997), pp.498-502.

[10] M. Otsuka, H. Maehara, M. Souli and S. Itoh; Study on development of vessel for shock pressure treatment for food, The International Journal of Multiphysics, Vol.1, No.1 (2007), pp.69-84.

[11] A. Osada, H. Hamashima, Y. Kato and S. Itoh; Study on low velocity detonation phenomena in nitromethane, The International Journal of Multiphysics, Vol.3, No.1 (2009), pp.1-10.

[12] S. Irie, K. Greg, Z. Ren and S. Itoh; Dynamic property of aluminum foam, The International Journal of Multiphysics, Vol.4, No.2 (2010), pp.103-111.

[13] O. Higa, A. Yasuda, Y. Higa, K. Shimojima, K. Hokamoto and S. Itoh; Optical Examination of Shockwave Propagation Induced by an Underwater Wire Explosion, The International Journal of Multiphysics, Vol.10, No.4 (2016), pp.343-353.

[14] P.R. Berventon; Data Reduction and Error Analysis for Physical Sciences, McGraw Hill, New York, (1969).

[15] H. Hornberg; Determination of Fume State Parameters from Expansion Measurements of Metal Tubes, Propellants, Explosives, Pyrotechnics, Vol.11, No.1 (1986), pp.23-31.

[16] C.W. Hirt, A.A. Amsden and J.L. Cook; An Arbitrary Lagrangian Eulerian Computing Method for All Flow Speeds, J. Computational Physics Vol.14, No.3 (1974), pp.227253.

[17] C.L. Mader; Numerical Modeling of Detonations, University of California Press (1979).

[18] RADIOSS theory manual 14.0 version, Large Displacement Finite Element Analysis, Altair Eng., Inc. (2015)

[19] S.P. Marsh; LASL Shock Hugoniot Data, University of California Press, Berkeley (1980). 
